\title{
Multimodality Imaging of Charcoal Granuloma Mimicking Breast
}

\section{Cancer Recurrence}

\author{
Ji Eun Shin, ${ }^{1}$ Jung Yin Huh, ${ }^{1,}{ }^{*}$ Hai-Lin Park, ${ }^{2}$ and Song Mi Noh ${ }^{3}$ \\ ${ }^{1}$ Department of Radiology, Gangnam CHA Hospital, CHA University College of Medicine, Seoul, Korea \\ ${ }^{2}$ Department of Surgery, Gangnam CHA Hospital, CHA University College of Medicine, Seoul, Korea \\ ${ }^{3}$ Department of Pathology, Gangnam CHA Hospital, CHA University College of Medicine, Seoul, Korea \\ "Corresponding author: Jung Yin Huh, Department of Radiology, Gangnam CHA Hospital, 566 Nonhyeon-ro, Gangnam-gu, CHA University College of Medicine, Seoul, Korea. \\ Tel: +82-234683214, Fax: +82-234683087, E-mail: physicomed@hanmail.net
}

Received 2017 February 18; Revised 2017 October 18; Accepted 2017 October 28.

\begin{abstract}
Charcoal is a biologically inert material used for the preoperative localization of nonpalpable breast lesions; its foreign body reactions have occasionally been reported. Although a few case reports on the formation of charcoal granulomas in the breast because of such reactions exist, to the best of our knowledge, no report includes breast-imaging findings obtained using multiple modalities. Here we report a case of two charcoal granulomas that mimicked breast cancer recurrence and provide breast-imaging findings obtained using mammography, ultrasonography, magnetic resonance imaging, and positron emission tomography/computed tomography. We also discuss the radiological and pathological features of charcoal granulomas. Awareness of breast imaging findings using multiple modalities along with the clinical and pathological features of charcoal granulomas helps in the differential diagnoses of newly detected lesions on postoperative follow-ups and reduces the need of unnecessary invasive procedures.
\end{abstract}

Keywords: Breast, Charcoal, Granuloma, Mammography, Ultrasonography, Magnetic Resonance Imaging

\section{Introduction}

Foreign-body granulomas in the breast are uncommon. However, they may result from various causes, including residual suture materials, silicone injections, gunshot wounds, and charcoal-suspension injections for preoperative localization of nonpalpable lesions (1-5). Generally, charcoal is a stable material that does not trigger foreign-body reactions; however, it may cause granulomatous reactions if it remains in situ for $>6$ months (6). Although some cases of foreign-body reactions to charcoalsuspension injections have been reported, to the best of our knowledge, their findings on various breast-imaging studies (e.g., mammography, ultrasonography (US), magnetic resonance imaging (MRI), positron emission tomography/computed tomography (PET/CT)) have not yet been reported. We report here a case of charcoal granulomas mimicking breast cancer recurrence in postoperative follow-up imaging studies in a breast cancer patient and discuss the radiological and pathological features of foreign-body granulomas caused by charcoal-suspension injections.

\section{Case Presentation}

A 49-year-old woman was referred to our institution with lesions in her left breast. She had undergone vacuumassisted biopsy (VAB) at two sites in her left breast 1 week earlier at an outside clinic. One lesion was confirmed as ductal carcinoma in situ with suspicious invasion. The patient had no family history of breast cancer and no history of other risk factors. Mammography and US were performed at our institution. The mammography revealed two oval, circumscribed, isodense masses surrounded by several microcalcifications in the upper outer quadrant of the left breast. Two corresponding cystic lesions were observed on US, suggestive of post-VAB changes with uncertainty for remnant cancer. Preoperative breast MRI revealed peripheral heterogeneous enhancement around both biopsied sites. Although these were considered parenchymal changes after $\mathrm{VAB}$, the possibility of residual cancer around the 2 VAB sites could not be excluded. Finally, the patient underwent breast-conserving surgery (BCS) after US-guided skin marking in the left breast.

On multiple cut sections obtained from BCS, the two previous VAB defects were noted to be filled with hemorrhagic material. The surrounding parenchyma contained 
fibrotic focal granular areas, but no definite mass was detected. Although no residual tumor cells were detected microscopically, two atypical ductal hyperplasia foci were located near the surgical margin (within $0.1 \mathrm{~mm}$ ). On postoperative follow-up mammography and US at 6 months, a 0.9$\mathrm{cm}$ mass was noted near the BCS site. This mass appeared as a circumscribed, iso-density lesion on mammography, whereas it appeared as a hyperechoic mass with marked posterior shadowing on US. It was considered to be a postoperative change and was categorized as breast imagingreporting and data system (BI-RADS) category 3 (probably benign finding).

One year after BCS, the patient underwent 18Ffluorodeoxyglucose (FDG)-PET/CT because she was anxious about cancer. FDG-PET/CT revealed two focal areas of FDG uptake with maximum standardized uptake values of 2.9 in the upper outer quadrant of the left breast, adjacent to the operative site (Figure 1). The FDG-PET/CT report noted the possibility of cancer recurrence.

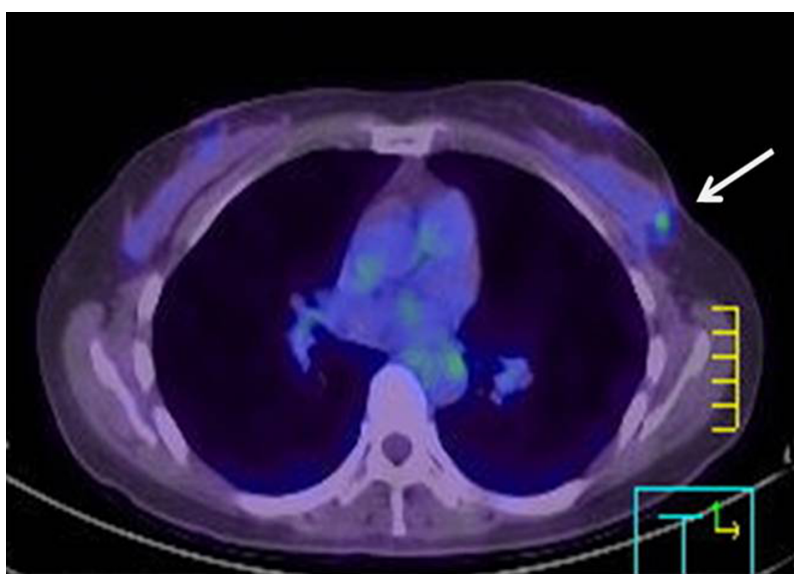

Figure 1. A 50-year-old woman at one-year follow-up after breast conserving surgery. Axial fused 18F-fluorodeoxyglucose positron-emission tomography/computed tomography image shows increased tracer uptake in the left breast (arrow).

Mammography and US were subsequently performed. Mammography demonstrated the previously detected mass and another newly developed mass near the previous operative site (Figure 2). On US, the previously detected mass had increased in size from $0.9 \mathrm{~cm}$ to 1.5 $\mathrm{cm}$, and the new mass measured $0.7 \mathrm{~cm}$ in its largest diameter. Both masses showed similar features on mammography and US. These hyperechoic masses had indistinct margins, markedly hypoechoic posterior shadowing, and increased vascularity at the junction of the breast parenchyma and subcutaneous fat layers (Figure 3). Although the US findings of hyperechoic masses favored postoperative changes, such as fat necrosis, the evidence of combined vascularity, increased size and iso-density on mammography implied that we could not exclude local recurrence despite the fact that radiological appearances of fat necrosis are variable. Furthermore, atypical ductal hyperplasia had been reported near $(<0.1 \mathrm{~mm})$ the resection margin of the breast-conserving surgery (BCS) specimen according to the pathological report.

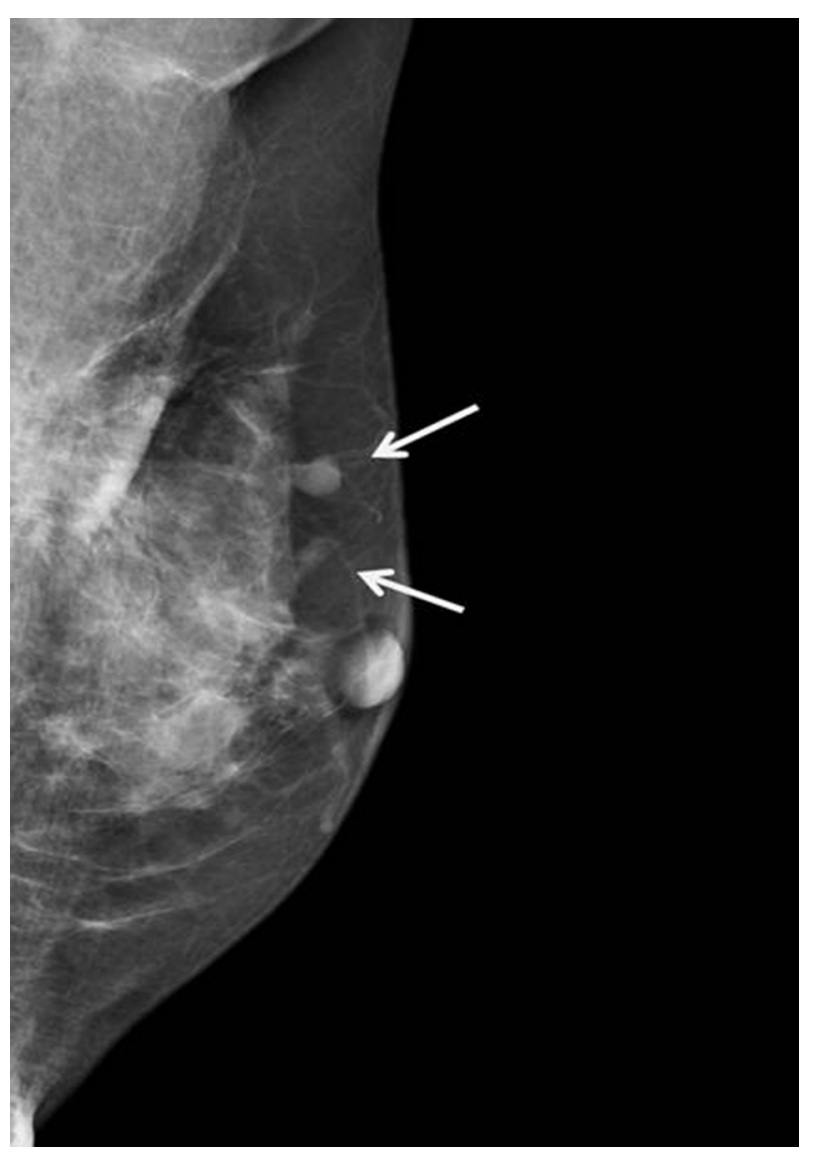

Figure 2. Left mediolateral oblique mammogram showing two masses of equal density with circumscribed margins in the left upper outer quadrant of the breast (arrows).

Breast MRI was requested to investigate the two possible local recurrences reported on PET/CT before biopsy. MRI revealed two circumscribed masses with iso-signal intensity on pre-contrast T1-weighted and T2-weighted images, and an initial rapid enhancement and plateau pattern on dynamic contrast-enhanced sequences (Figure 4).

Next, the two new masses were subjected to US-guided VAB with an 11-gauge needle (Mammotome ${ }^{\circledR}$; Devicor Medical Products, Inc., Cincinnati, OH, USA). Pathologically, the biopsy revealed giant cells, granular black pigment, and histiocytes (Figure 5) without evidence of malignancy. The pathological diagnosis was consistent with foreign-body 

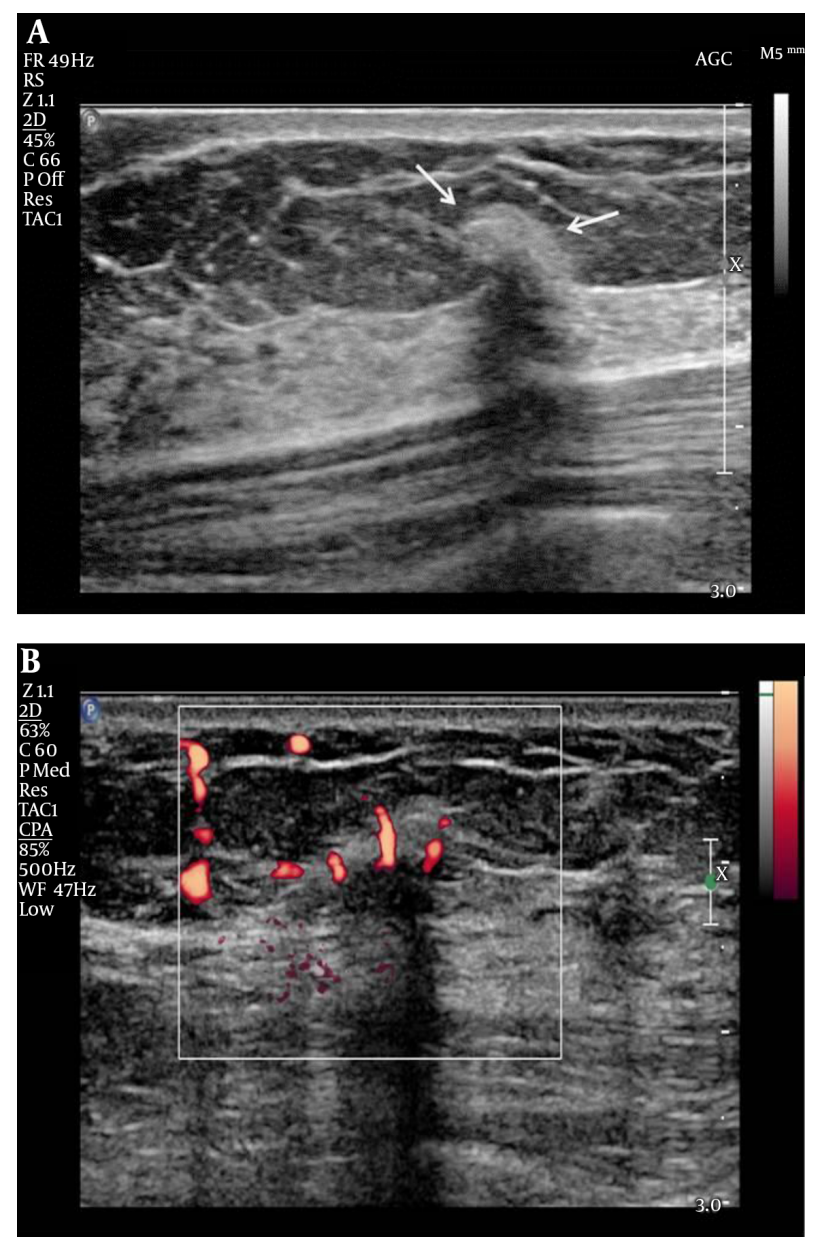

Figure 3. A, Longitudinal ultrasonography shows an indistinct, hyperechoic mass with posterior shadowing; this $1.5 \mathrm{~cm}$ diameter mass was observed at the 1 o'clock position in the left breast (arrows). B, Color Doppler sonography reveals increased vascularity surrounding the lesion.

granulomas caused by charcoal pigment.

We discussed the pathological results with the patient's surgeon and learned that during BCS, the surgeon had intraoperatively administered US-guided charcoalsuspension injections at the two sites where previous VABs had been performed at the outside clinic. Usually, charcoal localization is performed by breast radiologists at our institution, but this has been the only case intraoperatively performed by a breast surgeon without radiologists. If the radiologists had known about the use of charcoal for intraoperative localization, interpretation might have favored a benign diagnosis. Nevertheless, the complicated management of the patient allowed us to obtain multimodal imaging findings that provide valuable information when assessing charcoal granulomas that mimic breast cancer recurrence.

\section{Discussion}

Breast-screening US has increased the detection rate of nonpalpable breast cancer, and BCS has become the treatment of choice for early breast cancer $(7,8)$. Adequate preoperative localization is very important for the successful and minimal excision of a nonpalpable breast lesion. Several procedures such as wire-guided localization, radioisotope localization, dye injection (toluidine blue or methylene blue), and charcoal marking have been used to localize nonpalpable breast lesions (7-10). Compared to other procedures, charcoal marking avoids the risk of displacement and can thus be used with delayed surgeries (7).

Charcoal is a biologically inert, ubiquitous, exogenous pigment, and charcoal particles ingested by macrophages cause little or no inflammation (1). Generally, charcoal remains stable and does not trigger a foreign-body reaction; however, if it remains in situ for $>6$ months, it may cause a granulomatous reaction (6). In both in vivo and in vitro studies, charcoal is removed from the injection site at a very slow rate and therefore may cause inflammatory processes and fibroplastic responses (11). These low-grade foreign-body reactions can mimic malignancy.

A few cases of charcoal granuloma in the breast have been reported to date. Patrikeos et al. (1) reported three cases of carbon granulomas that developed after carbontrack localization. Ruiz-Delgado et al. (2) described 130 breast lesions subjected to VAB with carbon marking of the biopsy site; of these cases, four showed foreign-body giantcell reactions. However, to our knowledge, none of the previous reports described the multimodal imaging findings of foreign-body granulomas that arose after charcoal injection.

In our case, circumscribed margins and iso-density masses were observed via mammography. According to previous studies, charcoal granulomas are iso to highdensity lesions that have well-circumscribed or speculated margins $(1,2)$. US revealed indistinct margins and hyperechoic masses with marked posterior shadowing, which are unusual findings even for malignancies. A previous study of charcoal granulomas showed hypo-echogenicity, indistinct margins, and posterior shadowing on US (2). In contrast, Choi et al. (6) reported that a charcoal granuloma had shifted from hyperechoic to hypoechoic over time. The mammography and US features of our case are in accordance with those of previous reports and thus might be helpful for differentiating a recurrent tumor.

On MRI, iso-signal intensity in the lesions on precontrast T1- and T2-weighted images and rapid initial enhancement are relatively typical findings of cancer. However, the lesions in this case showed a plateau pattern on the kinetic curve and a centrifugal enhancement pattern 

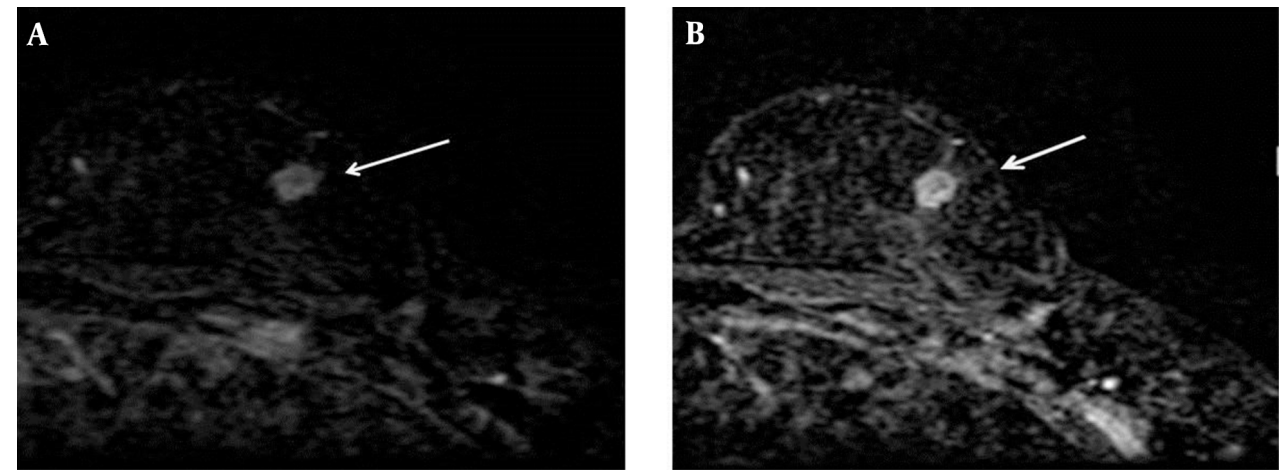

C

C Time Graph

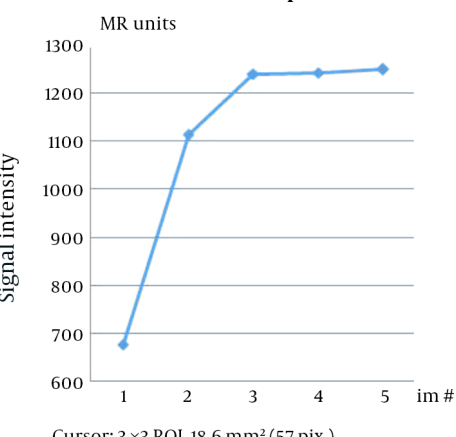

Figure 4. A, Axial subtracted T1-weighted magnetic resonance image obtained 1 min after gadolinium enhancement demonstrates a mass with a circumscribed margin and rapid enhancement in the left breast (arrow). B and C, In a delayed phase image, the mass shows peripheral enhancement and plateau curve pattern.

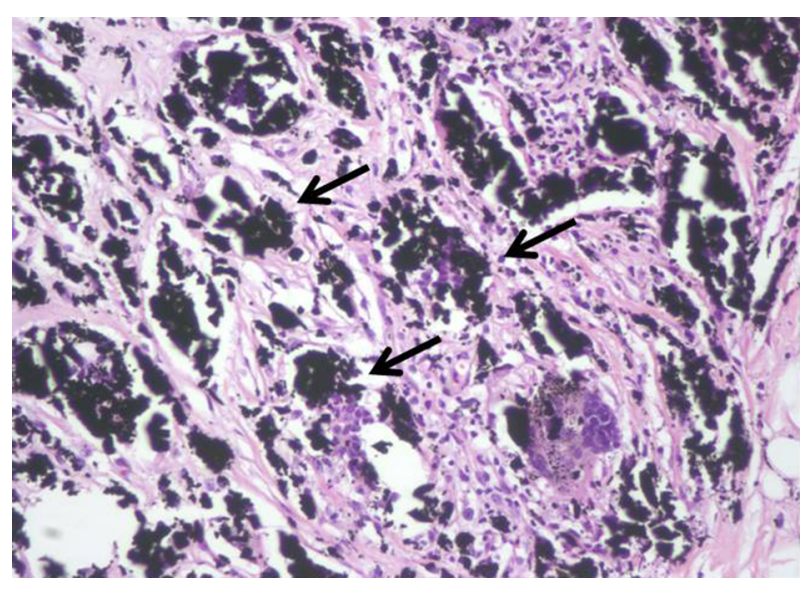

Figure 5. Photomicrograph of a histological specimen reveals numerous areas of black pigmentation suggestive of charcoal deposition (arrows), giant cells, and fibrosis (hematoxylin and eosin, magnification $\times 200$ ).

in the delayed phase. These are not typical imaging findings of malignancy, because breast cancer tends to en- hance from the periphery to the center (12). The imaging findings in our case might be explained by the development of fibrosis surrounded by blood vessels. FDG-PET/CT can help to detect a local recurrence or distant metastasis of breast cancer. Nevertheless, several benign lesions, such as acute and chronic inflammatory lesions and benign focal breast masses (e.g., silicone granuloma, fat necrosis, and postsurgical changes) may show increased FDG uptake on PET/CT (13). Charcoal granulomas can also show hypermetabolism (6). In this case, activated multinucleated giant cells utilized glucose for granuloma formation.

Several reasons justified further investigation in this case. First, atypical ductal hyperplasia was microscopically noted close to the surgical margin. Second, the radiologist was unaware of the history of intraoperative charcoal injection. Third, the imaging findings were inconsistent with typical postoperative changes and the lesion had also gradually increased in size. Together, those findings suggested the possibility of a recurrent lesion. However, knowledge of the patient's history and potential foreignbody reaction would have led to the inclusion of charcoal granuloma in the differential diagnosis. 
In conclusion, charcoal granulomas appeared as circumscribed, equal-density masses on mammography; hyperechogenic areas with posterior acoustic shadowing and increased vascularity on US; lesions with centrifugal enhancement on MRI; and hypermetabolic lesions on FDGPET/CT. Consideration of these findings and inclusion of charcoal granulomas in the differential diagnosis could decrease the use of unnecessary invasive procedures in similar cases in the future.

\section{Footnotes}

Authors' Contributions: Ji Eun Shin and Jung Yin Huh developed the original idea, case review, analysis and drafted the manuscript. Hai-Lin Park, Song Mi Noh contributed to the abstracted data, and prepared the manuscript. All authors read and approved the final manuscript.

Financial Disclosure: Authors had no financial interests related to the material in the manuscript.

Funding/Support: Authors received no funding related to this manuscript.

\section{References}

1. Patrikeos A, Wylie EJ, Bourke A, Frost F. Imaging of carbon granulomas of the breast following carbon track localization. Clin $\mathrm{Ra}$ diol. 1998;53(11):845-8. doi: 10.1016/S0009-9260(98)80198-0. [PubMed: 9833790].

2. Ruiz-Delgado ML, Lopez-Ruiz JA, Saiz-Lopez A. Abnormal mammography and sonography associated with foreign-body giant-cell reaction after stereotactic vacuum-assisted breast biopsy with carbon marking. Acta Radiol. 2008;49(10):1112-8. doi: 10.1080/02841850802452075. [PubMed: 18932053].
3. Shepherd SM, Makariou E. Silicone granuloma mimicking breast cancer recurrence on PET CT. Breast J. 2010;16(5):551-3. doi: 10.1111/j.15244741.2010.00948.x. [PubMed: 20604798].

4. Wakabayashi M, Reid JD, Bhattacharjee M. Foreign body granuloma caused by prior gunshot wound mimicking malignant breast mass. AJR Am J Roentgenol. 1999;173(2):321-2. doi: 10.2214/ajr.173.2.10430127. [PubMed: 10430127].

5. Yazici B, Sever AR, Mills P, Fish D, Jones S, Jones P. A rare cause of breast mass that mimics carcinoma: Foreign body reaction to amorphous surgical material. Wien Klin Wochenschr. 2007;119(15-16):497-9. doi: 10.1007/s00508-007-0835-y. [PubMed: 17721770].

6. Choi JW, Moon WJ, Choi N, Roh HG, Kim MY, Kim NR, et al. Charcoalinduced granuloma that mimicked a nodal metastasis on ultrasonography and FDG-PET/CT after neck dissection. Korean J Radiol. 2015;16(1):196-200. doi: 10.3348/kjr.2015.16.1.196. [PubMed: 25598690].

7. Corsi F, Sorrentino L, Bossi D, Sartani A, Foschi D. Preoperative localization and surgical margins in conservative breast surgery. Int J Surg Oncol. 2013;2013:793819. doi: 10.1155/2013/793819. [PubMed: 23986868].

8. Ko K, Han BK, Jang KM, Choe YH, Shin JH, Yang JH, et al. The value of ultrasound-guided tattooing localization of nonpalpable breast lesions. Korean J Radiol. 2007;8(4):295-301. doi:10.3348/kjr.2007.8.4.295. [PubMed: 17673840].

9. Czarnecki DJ, Feider HK, Splittgerber GF. Toluidine blue dye as a breast localization marker. AJR Am J Roentgenol.1989;153(2):261-3. doi: 10.2214/ajr.153.2.261. [PubMed: 2750608].

10. Rampaul RS, MacMillan RD, Evans AJ. Intraductal injection of the breast: a potential pitfall of radioisotope occult lesion localization. Br J Radiol. 2003;76(906):425-6. doi: 10.1259/bjr/27639166. [PubMed: 12814931].

11. Bonhomme-Faivre L, Depraetere P, Savelli MP, Amdidouche D, Bizi E, Seiller M, et al. Charcoal suspension for tumor labelling modifies macrophage activity in mice. Life Sci. 2000;66(9):817-27. doi: 10.1016/S0024-3205(99)00654-2. [PubMed: 10698356].

12. Han BK, Choe YH, Ko YH, Nam SJ, Yang JH. Foreign body granulomas of the breast presenting as bilateral spiculated masses. Korean J Radiol. 2001;2(2):113-6. doi: 10.3348/kjr.2001.2.2.113. [PubMed: 11752980].

13. Adejolu M, Huo L, Rohren E, Santiago L, Yang WT. False-positive lesions mimicking breast cancer on FDG PET and PET/CT. AJR Am J Roentgenol. 2012;198(3):W304-14. doi: 10.2214/AJR.11.7130. [PubMed: 22358030]. 